\title{
The energy release rate and the J-integral of an electrically insulated crack in a piezoelectric material
}

\author{
Cun-Fa Gao, Minghao Zhao, Pin Tong and Tong-Yi Zhang* \\ Department of Mechanical Engineering, Hong Kong University of Science and Technology \\ Clear Water Bay, Kowloon, Hong Kong, China
}

\begin{abstract}
In this work, we examine the energy release rate and the J-integral of an electrically insulated crack in a piezoelectric solid under remotely uniform electrical/mechanical loads. We model the crack as a slender elliptical flaw to take into account the electric field inside the crack and obtain the exact and explicit solution. The analytic results show that the energy release rate, in general, does not equal to the J-integral and that the J-integral is path-dependent due to the electric traction on the crack faces. However, the energy release rate is equal to the sum of the J-integral along an infinitesimal circle around the crack tip and the average driving force produced by the electric field on the entire crack surface. Under combined mechanical and electrical loading, the energy release rate may be positive or negative, depending on the level of the mechanical load and the strength of the electric field as well. The effect of electric field on the fracture behavior is mechanical load-dependent, which has been observed in experiments.
\end{abstract}

Keywords: Piezoelectric material, Electrically insulated crack, Energy release rate, J-integral.

\footnotetext{
* Corresponding author, Tel. +852-2358-7193, Fax +852-2358-1543, E-mail: mezhangt@ust.hk
} 


\section{Introduction}

Fracture of piezoelectric materials has been of considerable interest in recent decades. One can cite the work of Parton [1], Deeg [2], Pak [3], Sosa [4], Zhang and Hack [5], Suo et al. [6], Park and Sun [7], Gao and Barnett [8], Zhang and Tong [9], Zhang et al. [10], Ru [11], Gao and Fan [12], McMeeking [13, 14], and others. Recently, an extensive summary of the previous work was presented in [15]. However, it should be noted that nearly all previous works focus mainly on mathematical cracks without an initial crack opening. In fact, a real physical crack has a finite width. In 1989, McMeeking [16] modeled a crack in an isotropic dielectric material as a sharp elliptical flaw with a low permittivity. He found that the crack-tip field was dependent on the ratio: $(b / a) /\left(\varepsilon_{f} / \varepsilon_{m}\right)$, where $\varepsilon_{f}$ and $\varepsilon_{m}$ are the permittivities of the flaw and the matrix, respectively; and $a$ and $b$ are the major and minor semi-axes of the elliptical flaw, respectively. McMeeking [16] pointed out that neglecting the electric field inside the crack might lead to an erroneous estimate of crack-tip fields. Zhang and Tong [9], and Zhang et al. [10] further developed McMeeking's work [16] for piezoelectric media by introducing a similar ratio defined as $\lambda=(b / a) /\left(\varepsilon_{f} / \varepsilon_{\text {eff }}\right)$, where $\varepsilon_{\text {eff }}$ is an effective permittivity of the piezoelectric matrix. We call $\lambda$ the electrical impermeability of the crack because the higher the ratio is, the more impermeable the crack will be. Typically, a crack can be classified into three types, i.e., permeable crack $(\lambda=0)$, semi- permeable $\operatorname{crack}(\lambda=\mathrm{a}$ nonzero finite value), and impermeable crack $(\lambda \rightarrow \infty)[9,10]$. In fracture mechanics analysis, the permeable crack model is equivalent to letting $b=0$, while the impermeable crack model is equivalent to taking $\varepsilon_{f}=0$. In addition to the ratio $\lambda$, Zhang [17] introduced the ratio of $Z=(h / H) /\left(\varepsilon_{f} / \varepsilon_{\text {eff }}\right)$, where $h$ and $H$ denote the sizes of the crack width and the specimen, 
respectively. The results of a simple capacitor-model indicate that the ratio $\mathrm{Z}$ functions similarly to the ratio $\lambda$ and influences the energy release rate. McMeeking [13] also analyzed the energy release rate with a capacitor crack model, in which a mechanical load opens the crack, and obtained the similar conclusion that the crack opening (width) plays an important role in the energy release rate. More recently, Li [18] modeled a mode-III crack as a rectangular slit with an infinitesimal width in a piezoelectric solid, and calculated the local and global energy release rates. McMeeking [19] further studied the energy release rate of a general mode crack, modeled as a sharp elliptical cavity, in an infinite piezoelectric material. The energy change due to the introduction of the cavity is determined by the Eshelby inclusion technique and then the energy release rate is calculated. The results $[18,19]$ show that the initial opening of the crack may play an important role in crack propagation, which has been confirmed by a recent experimental observation [20].

In the present work we study a generalized $2 \mathrm{D}$ problem of a crack in a piezoelectric solid. The crack is treated as a slender elliptical flaw with a minor semi-axis $b=\delta_{c}$ and a major semi-axis $a$. We let $\delta_{c} \rightarrow 0$ while holding $\lambda$ finite and nonzero. The present approach is similar to McMeeking's work [19], in which the traditional energy balance approach is used to derive an explicit expression for the energy release rate of an electrically insulated crack. However, we place our emphasis on the J-integral and on the relationship between the energy release rate and the Jintegral. Below is the outline of the present paper: Section 2 gives the basic equations, and Section 3 lists the explicit results of an infinite piezoelectric solid with a sharp elliptical cavity with an electric field inside the cavity and complex potentials in the material. From the viewpoint of the traditional energy balance, the energy release rate $\mathrm{G}$ is derived in Section 4, and the relationship between the $\mathrm{G}$ and the J-integral is established in Section 5. As an application of the obtained results above, we 
discuss in details the effect of electric loading on piezoelectric fracture in Section 6. Finally, Section 7 concludes the work.

\section{Basic Equations}

In a rectangular coordinate system $x_{i}(\mathrm{i}=1,2,3)$, the basic equations for a linear piezoelectric solid are [21]

$$
\begin{gathered}
\sigma_{i j . j}=0, D_{i, i}=0, \\
\gamma_{i j}=\frac{1}{2}\left(u_{i, j}+u_{j, i}\right), E_{i}=-\varphi_{, i}, \\
\sigma_{i j}=C_{i j k l} \gamma_{k l}-e_{k i j} E_{k}, D_{k}=e_{k i j} \gamma_{i j}+\varepsilon_{k l} E_{l},
\end{gathered}
$$

where $u_{i}, \varphi, \sigma_{i j}, \gamma_{i j}, D_{j}$ and $E_{i}$ are the displacement, the electric potential, the stress, the strain, the electric displacement and the electric field, respectively, and $C_{i j k l}, e_{i j k}$ and $\varepsilon_{i j}$ denote the elastic, the piezoelectric and the dielectric constants, respectively. Consider a generalized two-dimensional problem, in which all field variables are independent of $x_{3}$. We introduce a generalized displacement vector $\mathbf{u}$ as $[15,21]$

$$
\mathbf{u}=\left[u_{1}, u_{2}, u_{3}, \varphi\right]^{T}=\mathbf{a} f\left(x_{1}+p x_{2}\right),
$$

where the superscript $T$ represents the transpose, $f\left(x_{1}+p x_{2}\right)$ is an analytic function, $p$ is a complex number, and a a constant four-element column vector. Equations (1), (2) and (3) can be satisfied by (4) for arbitrary $f\left(x_{1}+p x_{2}\right)$ if

$$
\left[\mathbf{Q}+p\left(\mathbf{R}+\mathbf{R}^{\mathbf{T}}\right)+p^{2} \mathbf{T}\right] \mathbf{a}=\mathbf{0},
$$

where the matrices $\mathbf{Q}, \mathbf{R}$ and $\mathbf{T}$ are given by 


$$
\mathbf{Q}=\left[\begin{array}{cc}
C_{i 1 k 1} & e_{11 i} \\
e_{11 i}^{T} & -\varepsilon_{11}
\end{array}\right], \mathbf{R}=\left[\begin{array}{cc}
C_{i 1 k 2} & e_{21 i} \\
e_{12 i}^{T} & -\varepsilon_{12}
\end{array}\right], \mathbf{T}=\left[\begin{array}{cc}
C_{i 2 k 2} & e_{22 i} \\
e_{22 i}^{T} & -\varepsilon_{22}
\end{array}\right], i, k=1,2,3
$$

The condition for nontrivial solutions to (5) requires

$$
\left|\mathbf{Q}+p\left(\mathbf{R}+\mathbf{R}^{\mathbf{T}}\right)+p^{2} \mathbf{T}\right|=0 .
$$

It can be shown that (6) has eight roots $p_{\alpha}$ and $\bar{p}_{\alpha}(\alpha=1,2,3,4)$ where $p_{\alpha}$ cannot be real because of the positive definiteness of the strain energy and electric energy densities. It is convenient to calculate $p_{\alpha}$ by solving the following standard eigen-equation:

$$
\mathbf{N} \zeta=p \zeta
$$

where

$$
\begin{gathered}
\mathbf{N}=\left(\begin{array}{ll}
\mathbf{N}_{1} & \mathbf{N}_{2} \\
\mathbf{N}_{3} & \mathbf{N}_{1}^{T}
\end{array}\right), \zeta=\left(\begin{array}{l}
\mathbf{a} \\
\mathbf{b}
\end{array}\right), \\
\mathbf{N}_{1}=-\mathbf{T}^{-1} \mathbf{R}^{T}, \mathbf{N}_{2}=\mathbf{T}^{-1}=\mathbf{N}_{2}^{T}, \mathbf{N}_{3}=\mathbf{R} \mathbf{T}^{-1} \mathbf{R}^{T}-\mathbf{Q}=\mathbf{N}_{\mathbf{3}}^{T} \text { and } \mathbf{b}=\left(\mathbf{R}^{T}+p \mathbf{T}\right) \mathbf{a} .
\end{gathered}
$$

The general solution of (1), (2) and (3) can be expressed as

$$
\begin{gathered}
\mathbf{u}=\mathbf{A f}\left(z_{*}\right)+\overline{\mathbf{A}} \overline{\mathbf{f}\left(z_{*}\right)}, \\
\phi=\mathbf{B f}\left(z_{*}\right)+\overline{\mathbf{B}} \overline{\mathbf{f}\left(z_{*}\right)},
\end{gathered}
$$

where

$$
\begin{gathered}
\mathbf{A}=\left(\mathbf{a}_{1}, \mathbf{a}_{2}, \mathbf{a}_{3}, \mathbf{a}_{4}\right), \mathbf{B}=\left(\mathbf{b}_{1}, \mathbf{b}_{2}, \mathbf{b}_{3}, \mathbf{b}_{4}\right), \\
\mathbf{f}\left(z_{*}\right)=\left[f_{1}\left(z_{1}\right), f_{2}\left(z_{2}\right), f_{3}\left(z_{3}\right), f_{4}\left(z_{4}\right)\right]^{T}, z_{\alpha}=x_{1}+p_{\alpha} x_{2},
\end{gathered}
$$

and $\phi$ is the generalized stress function such that

$$
\boldsymbol{\sigma}_{1}=\left[\sigma_{11}, \sigma_{12}, \sigma_{13}, D_{1}\right]^{T}=-\phi_{, 2},
$$




$$
\boldsymbol{\sigma}_{2}=\left[\sigma_{12}, \sigma_{22}, \sigma_{23}, D_{2}\right]^{T}=\phi_{, 1}
$$

\section{Field Intensity Factors for a Semi-Permeable Crack}

Consider an electrically insulated elliptical cavity of major semi-axis $a$ and minor semi-axis $b$ in an infinite piezoelectric medium subjected to the far-field mechanical/electric loading $\boldsymbol{\Sigma}_{2 \infty}=\left[\sigma_{21}^{\infty}, \sigma_{22}^{\infty}, \sigma_{23}^{\infty}, D_{2}^{\infty}\right]^{T}$ and $\boldsymbol{\Sigma}_{1 \infty}=\left[\sigma_{11}^{\infty}, \sigma_{12}^{\infty}, \sigma_{13}^{\infty}, D_{1}^{\infty}\right]^{T}$, as shown in Fig. 1. There is air or vacuum in the cavity with the dielectric constant $\varepsilon_{0}$ and the cavity surface is traction free. The solution in the cavity can be found in the literature, e.g., Zhang et al. [10], Gao and Fan [12], Gao [22], and others. Also according to these existing works, the complex function $\mathbf{f}\left(z_{*}\right)$ has the form

$$
\mathbf{f}\left(z_{*}\right)=\left\langle\left\langle z_{\alpha}\right\rangle\right\rangle \mathbf{c}^{\infty}+\mathbf{f}_{0}\left(z_{*}\right)
$$

where $\langle\langle\rangle\rangle$ denotes a diagonal matrix; $\mathbf{f}_{0}(z)$ is an unknown complex function, which is analytic at infinity, i.e., $\mathbf{f}_{0}(\infty)=\mathbf{0} ; \mathbf{c}^{\infty}$ is a constant related to the field variables at infinity such that

$$
\mathbf{c}^{\infty}=\mathbf{B}^{\mathrm{T}} \boldsymbol{\varepsilon}_{1 \infty}+\mathbf{A}^{\mathrm{T}} \boldsymbol{\Sigma}_{2 \infty}
$$

with

$$
\boldsymbol{\varepsilon}_{1 \infty}=\left[\varepsilon_{11}^{\infty}, \varepsilon_{12}^{\infty}+\omega_{3}^{\infty}, 2 \varepsilon_{13}^{\infty},-E_{1}^{\infty}\right]^{T} .
$$

Substituting (12) into (8) and (9) leads to

$$
\begin{aligned}
& \phi=\boldsymbol{\Sigma}_{2 \infty} x_{1}-\boldsymbol{\Sigma}_{1 \infty} x_{2}+2 \operatorname{Re}\left[\mathbf{K}_{0}\left(z_{*}\right)\right], \\
& \mathbf{u}=\boldsymbol{\varepsilon}_{1 \infty} x_{1}+\boldsymbol{\varepsilon}_{2 \infty} x_{2}+2 \operatorname{Im}\left[\mathbf{Y} \mathbf{K}_{0}\left(z_{*}\right)\right],
\end{aligned}
$$


where

$$
\boldsymbol{\varepsilon}_{2 \infty}=\left[\varepsilon_{21}^{\infty}-\omega_{3}^{\infty}, \varepsilon_{22}^{\infty}, 2 \varepsilon_{23}^{\infty},-E_{2}^{\infty}\right]^{T}, \mathbf{K}_{0}\left(z_{*}\right)=\mathbf{B} \mathbf{f}_{0}(z), \mathbf{Y}=i \mathbf{A B}^{-1}
$$

In the equations above, the generalized strain vectors $\boldsymbol{\varepsilon}_{1 \infty}$ and $\boldsymbol{\varepsilon}_{2 \infty}$ can be expressed in terms of $\sigma_{i j}^{\infty}$ and $D_{j}^{\infty}$ through the constitutive equations. Generally, the additional condition of zero rotation around the $x_{3}$-axis at infinity, i.e., $\omega_{3}^{\infty}=0$, can be imposed.

Under remotely uniform loading, the electric displacement inside the cavity is uniform with components $D_{2}^{0}$ and $D_{1}^{0}$. Thus, we can determine $\mathbf{K}_{0}\left(z_{*}\right)$ in (13) and (14) from the boundary condition on the cavity surface. The final result is [22]

$$
\mathbf{K}_{0}\left(z_{*}\right)=-\frac{1}{2}\left\langle\left\langle\zeta_{\alpha}^{-1}\left(z_{\alpha}\right)\right\rangle\right\rangle \mathbf{p}
$$

where

$$
\begin{gathered}
\zeta_{\alpha}^{-1}\left(z_{\alpha}\right)=\frac{z_{\alpha}-\sqrt{z_{\alpha}^{2}-c_{\alpha}^{2}}}{a+i p_{\alpha} b}, c_{\alpha}^{2}=a^{2}+p_{\alpha}^{2} b^{2}, \\
\mathbf{p}=\left[a \sigma_{21}^{\infty}-i b \sigma_{11}^{\infty}, a \sigma_{22}^{\infty}-i b \sigma_{12}^{\infty}, a \sigma_{23}^{\infty}-i b \sigma_{13}^{\infty}, a\left(D_{2}^{\infty}-D_{2}^{0}\right)-i b\left(D_{1}^{\infty}-D_{1}^{0}\right)\right]^{T}, \\
D_{2}^{\infty}-D_{2}^{0}=\frac{b\left(D_{2}^{\infty}-\varepsilon_{0} E_{2}^{\infty}\right)+\varepsilon_{0} \operatorname{Re} \sum_{j=1}^{3} Y_{4 j} M_{j}^{\infty}}{b-a \varepsilon_{0} Y_{44}}, \\
D_{1}^{\infty}-D_{1}^{0}=\frac{a\left(D_{1}^{\infty}-\varepsilon_{0} E_{1}^{\infty}\right)-\varepsilon_{0} \operatorname{Im} \sum_{j=1}^{3} Y_{4 j} M_{j}^{\infty}}{a-b \varepsilon_{0} Y_{44}} \\
\mathbf{M}^{\infty}=\left[a \sigma_{21}^{\infty}-i b \sigma_{11}^{\infty}, a \sigma_{22}^{\infty}-i b \sigma_{12}^{\infty}, a \sigma_{23}^{\infty}-i b \sigma_{13}^{\infty}\right]^{T} .
\end{gathered}
$$


For a slender elliptical flaw with $b=\delta_{c} \rightarrow 0, b \varepsilon_{0}$ is so small that all terms related to $b \varepsilon_{0}$ may be neglected in (18) and (19), which then can be approximated as

$$
\begin{gathered}
D_{2}^{\infty}-D_{2}^{0}=\frac{b D_{2}^{\infty}+a \operatorname{Re} \sum_{j=1}^{3} Y_{4 j} \sigma_{2 j}^{\infty}}{b-a \varepsilon_{0} Y_{44}}, \\
D_{1}^{\infty}-D_{1}^{0}=D_{1}^{\infty}-\varepsilon_{0} E_{1}^{\infty}-\varepsilon_{0} \operatorname{Im} \sum_{j=1}^{3} Y_{4 j} \sigma_{2 j}^{\infty} .
\end{gathered}
$$

From (21) and (22), we can gives the electric field inside the crack as

$$
\begin{aligned}
& D_{2}^{0}=D_{2}^{\infty}-\frac{\lambda D_{2}^{\infty}-\frac{1}{H_{44}} \sum_{j=1}^{3} H_{4 j} \sigma_{2 j}^{\infty}}{1+\lambda}, \\
& D_{1}^{0}=\varepsilon_{0} E_{1}^{\infty}+\varepsilon_{0} \sum_{j=1}^{3} \operatorname{Im}\left[Y_{4 j}\right] \sigma_{2 j}^{\infty},
\end{aligned}
$$

where $\mathbf{H}=\mathbf{Y}+\overline{\mathbf{Y}}$, and

$$
\lambda=\alpha / \beta, \alpha=\delta_{c} / a, \beta=\varepsilon_{0} / \varepsilon^{e f f}, \varepsilon^{e f f}=-Y_{44}^{-1} .
$$

For this case, the complex potential is still given by (15) with the simplified expressions of $\mathbf{p}$ and $\zeta_{\alpha}^{-1}\left(z_{\alpha}\right)$

$$
\begin{gathered}
\mathbf{p}_{c}=a \Pi_{2 \infty}, \Pi_{2 \infty}=\left[\sigma_{21}^{\infty}, \sigma_{22}^{\infty}, \sigma_{23}^{\infty}, D_{2}^{\infty}-D_{2}^{0}\right]^{T}, \\
\zeta_{\alpha}^{-1}\left(z_{\alpha}\right)=\frac{1}{a}\left(z_{\alpha}-\sqrt{z_{\alpha}^{2}-a^{2}}\right) .
\end{gathered}
$$

Then, then intensity factor vector takes the form

$$
\mathbf{k}=\sqrt{\pi a} \Pi_{2 \infty},
$$

where $D_{2}^{\infty}-D_{2}^{0}$ in $\Pi_{2 \infty}$ is determined from (23). 


\section{Energy Release Rate}

From the thermodynamic point of view, the energy release rate is the most appropriate physical quantity to characterize the fracture behavior. For purely elastic materials, the energy release rate $\mathrm{G}$ is identical to the J-integral because there is no energy stored in the crack cavity. For an electrically insulated crack in a piezoelectric material, however, a high electric field exists inside the crack. This electric energy can change the relationship between $G$ and J. In this section, we shall derive the exact expression of the energy release rate based on the principle of isothermal piezoelectric virtual work $[10,15]$.

There are four isothermal thermodynamic functions: the free energy, $f$, the electric enthalpy, $h$, the mechanical enthalpy, $w$, and the full Gibbs free energy, $g[10,15]$. Each of the four isothermal thermodynamic functions corresponds to an isothermal potential, $P$, which is defined as the difference between the generalized work, $W$, done by mechanical and electrical loads and the generalized mechanical and electrical energy, $U$, stored inside the body of interest. In the differential form, we have energy balance condition:

$$
\partial P=\partial W-\partial U
$$

Using the electric enthalpy $h$, the generalized work and the generalized stored energy are given by

$$
\begin{gathered}
W=\int_{\Gamma}\left(\sigma_{i j} n_{j} u_{i}+D_{i} n_{i} \varphi\right) d \Gamma, \\
U=\iint_{\Omega} h d \Omega,
\end{gathered}
$$


respectively, where $\Gamma$ denotes a contour as shown in Fig. 2, $\Omega$ is the integration domain enclosed by $\Gamma, u_{i}$ is the mechanical displacement, $D_{i} n_{i}$ is the prescribed electric displacement on $\Gamma$, and the electric enthalpy per unit volume $h$ takes the form:

$$
h=\frac{1}{2} \sigma_{i j} \varepsilon_{i j}-\frac{1}{2} D_{i} E_{i} .
$$

For 2D cracks, the energy release rate, $G$, for crack propagation can be defined as

$$
G=\frac{\partial P}{\partial a}=\frac{\partial}{\partial a}(W-U) .
$$

On the other hand, one has

$$
U=\iint_{\Omega} h d \Omega=\iint_{m} h d \Omega+\iint_{c} h d \Omega,
$$

where $m$ and $c$ stand for the material and the cavity, respectively. Using Green's theorem, we have

$$
\begin{gathered}
\iint_{m} h d \Omega=\int_{\Gamma} \frac{1}{2}\left(\sigma_{i j} n_{j} u_{i}+D_{i} n_{i} \varphi\right) d \Gamma+\oint_{\Gamma^{\prime}} \frac{1}{2}\left(D_{i} n_{i} \varphi\right) d \Gamma^{\prime}, \\
\iint_{c} h d \Pi=-\oint_{\Gamma^{\prime}} \frac{1}{2}\left(D_{i} n_{i} \varphi\right) d \Gamma^{\prime}
\end{gathered}
$$

where $\Gamma^{\prime}$ is the contour of the cavity as shown in Fig. 2. Substituting (34) and (35) in (33) results in

$$
U=\frac{1}{2} \int_{\Gamma}\left(\sigma_{i j} n_{j} u_{i}+D_{i} n_{i} \varphi\right) d \Gamma .
$$

Using (29) and (36), one obtains

$$
W-U=\frac{1}{2} \int_{\Gamma}\left(\sigma_{i j} n_{j} u_{i}+D_{i} n_{i} \varphi\right) d \Gamma .
$$

Substituting (37) into (32) gives

$$
G=\frac{1}{2} \int_{\Gamma}\left(\sigma_{i j} n_{j} \frac{\partial u_{i}}{\partial a}+D_{i} n_{i} \frac{\partial \varphi}{\partial a}\right) d \Gamma .
$$

Noting that $n_{3}=0$ on $\Gamma$, we have 


$$
\begin{aligned}
\sigma_{i j} n_{j} \frac{\partial u_{i}}{\partial a}+D_{i} n_{i} \frac{\partial \varphi}{\partial a} & =\left(\sigma_{11} \frac{\partial u_{1}}{\partial a}+\sigma_{21} \frac{\partial u_{2}}{\partial a}+\sigma_{31} \frac{\partial u_{2}}{\partial a}+D_{1} \frac{\partial \varphi}{\partial a}\right) n_{1} \\
& +\left(\sigma_{12} \frac{\partial u_{1}}{\partial a}+\sigma_{22} \frac{\partial u_{2}}{\partial a}+\sigma_{32} \frac{\partial u_{2}}{\partial a}+D_{2} \frac{\partial \varphi}{\partial a}\right) n_{2}=\boldsymbol{\sigma}_{1}^{T} \frac{\partial \mathbf{u}}{\partial a} n_{1}+\boldsymbol{\sigma}_{2}^{T} \frac{\partial \mathbf{u}}{\partial a} n_{2} .
\end{aligned}
$$

Using (10) and (11) in (39) gives

$$
\sigma_{i j} n_{j} \frac{\partial u_{i}}{\partial a}+D_{i} n_{i} \frac{\partial \varphi}{\partial a}=-\phi_{, 2}^{\mathrm{T}} \frac{\partial \mathbf{u}}{\partial a} n_{1}+\phi_{, 1}^{\mathrm{T}} \frac{\partial \mathbf{u}}{\partial a} n_{2}
$$

Due to

$$
n_{1} d \Gamma=d x_{2}, n_{2} d \Gamma=-d x_{1}
$$

we obtain from (40) and (41) that

$$
\begin{gathered}
\left(\sigma_{i j} n_{j} \frac{\partial u_{i}}{\partial a}+D_{i} n_{i} \frac{\partial \varphi}{\partial a}\right) d \Gamma=-\left[\phi_{, 2}^{\mathrm{T}} d x_{2}+\phi_{, 1}^{\mathrm{T}} d x_{1}\right] \cdot \frac{\partial \mathbf{u}}{\partial a} \\
=-\left[d \phi^{\mathrm{T}}\right] \cdot \frac{\partial \mathbf{u}}{\partial a}
\end{gathered}
$$

Inserting (42) into (38) leads to

$$
G=-\frac{1}{2} \int_{\Gamma}\left[d \phi^{\mathrm{T}}\right] \cdot \frac{\partial \mathbf{u}}{\partial a}
$$

From (14) one has

$$
\frac{\partial \mathbf{u}}{\partial a}=2 \operatorname{Im}\left[\mathbf{Y} \frac{\partial}{\partial a} \mathbf{K}_{0}\left(z_{*}\right)\right]
$$

If we take $\Gamma$ as a large elliptical contour enclosing the cavity, then

$$
x_{1}=a \rho_{0} \cos \theta, x_{2}=\delta_{c} \rho_{0} \sin \theta, \rho_{0}>>1,
$$

with $\rho \bar{\rho}=\rho_{0}^{2}$ and $\zeta_{\alpha}=\rho=\rho_{0} e^{i \theta}$ on $\Gamma$. One can write (45) as 


$$
x_{1}=\frac{1}{2} a\left(\frac{\rho_{0}^{2}}{\rho}+\rho\right), x_{2}=\frac{i}{2} \delta_{c}\left(\frac{\rho_{0}^{2}}{\rho}-\rho\right), \rho=\rho_{0} e^{i \theta} \text {, on } \Gamma \text {. }
$$

Moreover, one has from (15) that

$$
\mathbf{K}_{0}\left(z_{*}\right)=-\frac{1}{2 \rho} \mathbf{p}_{c} \text {, on } \Gamma
$$

Substituting (47) into (44) leads to

$$
\frac{\partial \mathbf{u}}{\partial a}=-\operatorname{Im}\left[\mathbf{Y} \frac{\partial \mathbf{p}_{c}}{\partial a} \frac{1}{\rho}\right]
$$

Equation (48) can be rewritten as

$$
\frac{\partial \mathbf{u}}{\partial a}=\mathbf{U}_{1} \rho+\mathbf{U}_{-1} \frac{1}{\rho}
$$

where

$$
\mathbf{U}_{1}=\frac{1}{2 \rho_{0}^{2}} \bar{i} \mathbf{Y} \frac{\partial \overline{\mathbf{p}}_{c}}{\partial a}, \mathbf{U}_{-1}=\frac{1}{2} i \mathbf{Y} \frac{\partial \mathbf{p}_{c}}{\partial a}
$$

A substitution of (46) and (47) into (13) yields

$$
\phi=\mathbf{W}_{1} \rho+\mathbf{W}_{-1} \rho^{-1},
$$

where

$$
\begin{aligned}
& \mathbf{W}_{1}=\frac{1}{2}\left(a \boldsymbol{\Sigma}_{2 \infty}+i \delta_{c} \boldsymbol{\Sigma}_{1 \infty}-\overline{\mathbf{p}}_{c} / \rho_{0}^{2}\right), \\
& \mathbf{W}_{-1}=\frac{1}{2}\left(a \boldsymbol{\Sigma}_{2 \infty} \rho_{0}^{2}-i \delta_{c} \boldsymbol{\Sigma}_{1 \infty} \rho_{0}^{2}-\mathbf{p}_{c}\right) .
\end{aligned}
$$

From (51) one has

$$
d \phi^{\mathrm{T}}=\left(\mathbf{W}_{1}^{T}-\mathbf{W}_{-1}^{T} \rho^{-2}\right) d \rho .
$$

Using (54) and (49), we obtain 


$$
\left[d \phi^{\mathrm{T}}\right] \cdot \frac{\partial \mathbf{u}}{\partial a}=\left(\mathbf{W}_{1}^{T}-\mathbf{W}_{-1}^{T} \rho^{-2}\right)\left(\mathbf{U}_{1} \rho+\mathbf{U}_{-1} \rho^{-1}\right) d \rho
$$

Substituting (55) into (43) leads to

$$
G=-\pi i\left(\mathbf{W}_{1}^{T} \mathbf{U}_{-1}-\mathbf{W}_{-1}^{T} \mathbf{U}_{1}\right)
$$

Finally, substituting (50), (52) and (53) into (56), and neglecting the terms of the order of $\rho_{0}^{-2}$ as $\rho_{0} \rightarrow \infty$, we find

$$
G=\frac{\pi a}{2} \operatorname{Im}\left[\left(\Sigma_{2 \infty}^{T}+i \Sigma_{1 \infty}^{T} \delta_{c} / a\right) i \mathbf{Y} \frac{\partial \mathbf{p}_{c}}{\partial a}\right]
$$

For $\delta_{c} / a \rightarrow 0,(57)$ becomes

$$
G=\frac{\pi a}{2} \operatorname{Im}\left[\Sigma_{2 \infty}^{T} i \mathbf{Y} \frac{\partial \mathbf{p}_{c}}{\partial a}\right]
$$

The term $\frac{\partial \mathbf{p}_{c}}{\partial a}$ can be determined from the first equation of (25). In the present work, we assume that the crack extends in a self-similar manner, that is, $b / a=a$ constant, which was adopted previously by Zhang et al. [10] and McMeeking [19]. It can be shown that this condition is equivalent to $D_{2}^{0}=$ a constant during crack propagation. With this assumption, we have

$$
\frac{\partial \mathbf{p}_{c}}{\partial a}=\left[\sigma_{21}^{\infty}, \sigma_{22}^{\infty}, \sigma_{23}^{\infty}, D_{2}^{\infty}-D_{2}^{0}\right]^{T}=\Pi_{2 \infty}
$$

Substituting (59) into (58), we obtain the expression for the energy release rate of a semi-permeable crack as

$$
G=\frac{\pi a}{4} \Sigma_{2 \infty}^{T} \mathbf{H} \Pi_{2 \infty}
$$

Since $\Sigma_{2 \infty}=\Pi_{2 \infty}+\mathbf{i}_{4} D_{2}^{0}$, where $\mathbf{i}_{4}=(0,0,0,1)^{T},(60)$ can be rewritten as

$$
G=\frac{\pi a}{4}\left(\Pi_{2 \infty}^{T}+\mathbf{i}_{4}^{T} D_{2}^{0}\right) \mathbf{H} \Pi_{2 \infty}
$$


that is

$$
G=\frac{\pi a}{4} \Pi_{2 \infty}^{T} \mathbf{H} \Pi_{2 \infty}+\frac{\pi a}{4} \mathbf{i}_{4}^{T} \mathbf{H} \Pi_{2 \infty} D_{2}^{0}
$$

Equation (61) can be further reduced to the form

$$
G=\frac{1}{4} \mathbf{k}^{T} \mathbf{H k}+\frac{\pi a}{4} \mathbf{i}_{4}^{T} \mathbf{H} \Pi_{2 \infty} D_{2}^{0}
$$

where $\mathbf{k}$ is the stress intensity vector defined in (27). Using the Eshelby inclusion technique, McMeeking [19] recently derived an expression of the energy release rate similar to (62). His equation (27) is identical (23) in the present work. However, there is a $\varepsilon_{0} E_{2}^{\infty}$ term in the first right term of his equation (26), which is neglected in his equation (27) and in the present work. Except of the $\varepsilon_{0} E_{2}^{\infty}$ term, McMeeking's result is the same as (62).

\section{Relationship between $G$ and the J-integral}

The J-integral in a piezoelectric solid can be defined as [23]

$$
J=\int_{\Gamma_{*}}\left(h n_{1}-\sigma_{i j} n_{j} u_{i, 1}-n_{i} D_{i} \varphi_{, 1}\right) d s,
$$

where $\Gamma_{*}$ is a contour enclosing the right crack tip, starting from a point on the lower crack surface and terminating at a point on the upper crack surface. Let us consider the J-integral of a closed contour $\Gamma_{0}=\Gamma_{\gamma}+\left(-\Gamma_{l}\right)+\left(-\Gamma_{c s}\right)$, where $\Gamma_{\gamma}$ is any contour integral starting at a point $x_{1}^{-}$on the lower crack surface and ending at the corresponding point $x_{1}^{+}$on the upper crack surface, $\Gamma_{l}$ is an infinitesimal circle of radius $r_{0}$ around the crack tip, and $\Gamma_{c s}$ represents parts of crack surfaces $\mathrm{AB}$ and $\mathrm{BC}$ as shown in Fig. 3. Since the J-integral for a closed contour is zero, we have 


$$
J_{0}=J_{\gamma}-J_{l}-\hat{J}_{c s}=0
$$

where $J_{l}$ may be called the local energy release rate along $\Gamma_{l}, J_{\gamma}$ is the J-integral along the contour $\Gamma_{\gamma}$, and

$$
\hat{J}_{c s}=J_{A B}+J_{B C}
$$

is the contribution from the electrical field along the crack surfaces $A B$ and BC. Using (63), we have

$$
J_{A B}=\int_{A B}\left(h+D_{1} E_{1}\right) n_{1} d s-\int_{A B} D_{2}^{0} \varphi_{, 1} n_{2} d s
$$

On the crack surface, $h+D_{1} E_{1}$ is free of singularities and $n_{1} \approx 0$ because $b=\delta_{c} \rightarrow 0$ and thus, the first integral in (66) can be neglected and (66) reduces to

$$
J_{A B}=D_{2}^{0}\left\lfloor\varphi^{-}\left(a^{*}\right)-\varphi^{-}\left(x_{1}\right)\right\rfloor,
$$

where $a^{*}=a-r_{0}$. Similarly, on the lower crack surface BA, one has

$$
J_{B C}=D_{2}^{0}\left\lfloor\varphi^{+}\left(x_{1}\right)-\varphi^{+}\left(a^{*}\right)\right\rfloor .
$$

Using (65), (67) and (68), we have

$$
\hat{J}_{c s}\left(x_{1}\right)=J_{A B}+J_{B C}=D_{2}^{0}\left[\varphi^{-}\left(a^{*}\right)-\varphi^{-}\left(x_{1}\right)\right]+D_{2}^{0}\left[\varphi^{+}\left(x_{1}\right)-\varphi^{+}\left(a^{*}\right)\right] .
$$

For $r_{0} \rightarrow 0, a^{*} \rightarrow a$ and $\varphi^{+}\left(a^{*}\right) \rightarrow \varphi^{-}\left(a^{*}\right),(69)$ approaches

$$
\hat{J}_{c s}\left(x_{1}\right)=D_{2}^{0}\left[\varphi^{+}\left(x_{1}\right)-\varphi^{-}\left(x_{1}\right)\right] \text {. }
$$

We can use the crack opening (14) to calculate the potential difference:

$$
\varphi^{+}\left(x_{1}, 0\right)-\varphi^{-}\left(x_{1}, 0\right)=2 \operatorname{Im}\left\langle\mathbf{Y}\left[\mathbf{K}_{0}^{+}\left(x_{1}, 0\right)-\mathbf{K}_{0}^{-}\left(x_{1}, 0\right)\right]\right\rangle_{4},
$$

where \langle\rangle$_{4}$ denotes the fourth row of the vector in \langle\rangle . Substituting (15) together with (25) and (26) into (71) yields 


$$
\varphi^{+}\left(x_{1}, 0\right)-\varphi^{-}\left(x_{1}, 0\right)=2 \operatorname{Im}\left\langle\mathbf{Y} i \Pi_{2 \infty}\right\rangle_{4} \sqrt{a^{2}-x_{1}^{2}}=\mathbf{i}_{4}^{T} \mathbf{H} \Pi_{2 \infty} \sqrt{a^{2}-x_{1}^{2}}, x_{1} \in[-a, a]
$$

Thus, (70) becomes

$$
\hat{J}_{c s}\left(x_{1}\right)=\mathbf{i}_{4}^{T} \mathbf{H} \Pi_{2 \infty} D_{2}^{0} \sqrt{a^{2}-x_{1}^{2}}, x_{1} \in[-a, a]
$$

Since $\hat{J}_{c s}\left(x_{1}\right)$ does not, in general, equal to zero, (64) indicates $J_{\gamma}-J_{l}=\hat{J}_{c s}\left(x_{1}\right) \neq 0$, and thereby shows that the integral $J_{\gamma}$ is path-dependent for an electrically insulated crack.

On the other hand, it can be shown from (63) that

$$
J_{l}=\frac{\pi a}{4} \Pi_{2 \infty}^{T} \mathbf{H} \Pi_{2 \infty}=\frac{1}{4} \mathbf{k}^{T} \mathbf{H} \mathbf{k}
$$

Then, (62) can be rewritten as

$$
G=J_{l}+\Delta G
$$

where $J_{l}$ is the local energy release rate and

$$
\Delta G=\frac{\pi a}{4} \mathbf{i}_{4}^{T} \mathbf{H} \Pi_{2 \infty} D_{2}^{0}
$$

To illustrate the physical meaning of $\Delta G$, we introduce the parameter $J_{c s}$ defined as

$$
J_{c s}=\frac{1}{a} \int_{0}^{a} \hat{J}_{c s}\left(x_{1}\right) d x_{1}
$$

which represents an average driving force produced by the electric field of the entire crack surfaces, as shown in Fig. 4. Substituting (73) into (77) and carrying out the integration, we find

$$
J_{c s}=\frac{\pi a}{4} \mathbf{i}_{4}^{T} \mathbf{H} \Pi_{2 \infty} D_{2}^{0}
$$

Comparing (78) and (76) gives

$$
\Delta G=J_{c s} .
$$


i.e., $\Delta G$ is the average contribution of the J-integral along the crack faces. This contribution is from the electric field within the crack because $J_{c s}$ involves only $D_{2}^{0}$. Substituting (79) into (75) gives

$$
G=J_{l}+J_{c s} .
$$

Equation (80) gives the relationship between the global energy release rate $G$ and the J-integrals, i.e., the global energy release rate equals to the sum of the local energy release rate $J_{l}$ and the average contribution of the J-integral along the crack faces. In other words, the J-integral $J_{l}$ is not, in general, equal to $G$ for an electrically insulated crack since $J_{c s} \neq 0$ and moreover the J-integral $J_{\gamma}$ is path-dependent.

Now, let us examine the J-integral along $\Gamma_{g}$, which starts at $x_{1}^{-}=0$ on the lower crack surface and ends at $x_{1}^{+}=0$ on the upper crack surface as shown in Fig. 3. Obviously the value of the Jintegral along $\Gamma_{g}$ can be determined from (64) as

$$
J_{g}=J_{l}+\hat{J}(0),
$$

where

$$
\hat{J}(0)=\hat{J}_{c s}(0)=a \mathbf{i}_{4}^{T} \mathbf{H} \Pi_{2 \infty} D_{2}^{0} .
$$

A substitution of (82) into (81) results in

$$
J_{g}=J_{l}+a \mathbf{i}_{4}^{T} \mathbf{H} \Pi_{2 \infty} D_{2}^{0} .
$$

Comparing (83) with (75) one finds $J_{g} \neq G$. This result is different from that of Li [18] who concluded that $J_{g}=G$ for a mode-III crack. This discrepancy might be resulted from the difference in the crack model. In Li's work, a crack is treated as a rectangular slit, while in the present work, a narrow elliptical cavity. In the present case, (83) and (75) give 


$$
J_{g}-G=0.215 a \mathbf{i}_{4}^{T} \mathbf{H} \Pi_{2 \infty} D_{2}^{0} .
$$

There are two extreme limits for an electrically insulated crack, i.e., electrically impermeable and permeable. For an impermeable crack, there is no electrical field inside the crack, i.e., $D_{2}^{0}=0$. Thus, $\hat{J}_{c s}\left(x_{1}\right)=J_{c s}=0$ and Eq. (80) reduces to

$$
G=J_{l}=\frac{1}{4} \mathbf{k}_{i m}^{T} \mathbf{H} \mathbf{k}_{i m} .
$$

where

$$
\mathbf{k}_{\text {im }}=\left[\sigma_{21}^{\infty}, \sigma_{22}^{\infty}, \sigma_{23}^{\infty}, D_{2}^{\infty}\right]^{T} \sqrt{\pi a},
$$

is the intensity factor vector of the impermeable crack. In this case, the energy release rate equals to the $\mathrm{J}$ integral and the $\mathrm{J}$ integral is path-independent.

For a permeable crack, $\lambda=0$ and (23) results in

$$
D_{2}^{\infty}-D_{2}^{0}=-\frac{1}{H_{44}} \sum_{j=1}^{3} H_{4 j} \sigma_{2 j}^{\infty},
$$

or

$$
\sum_{j=1}^{3} H_{4 j} \sigma_{2 j}^{\infty}+H_{44}\left(D_{2}^{\infty}-D_{2}^{0}\right)=0 .
$$

From the second equation of (25), Eq. (88) implies

$$
\mathbf{i}_{4}^{T} \mathbf{H} \Pi_{2 \infty}=0 .
$$

Substituting (89) into (73) and (78) yields

$$
\hat{J}_{c s}\left(x_{1}\right)=J_{c s}\left(x_{1}\right)=0 .
$$


Thus, (80) becomes

$$
G=J_{l}=\frac{1}{4} \mathbf{k}_{p}^{T} \mathbf{H k}_{p},
$$

where $\mathbf{k}_{p}$ is the intensity factor vector of the permeable crack given by

$$
\mathbf{k}_{p}=\left[\sigma_{21}^{\infty}, \sigma_{22}^{\infty}, \sigma_{23}^{\infty}, D_{2}^{\infty}-D_{2}^{0}\right]^{T} \sqrt{\pi a},
$$

in which $D_{2}^{\infty}-D_{2}^{0}$ is determined from (87). Equation (91) shows that for a permeable crack, the energy release rate also equals to the $\mathrm{J}$ integral and thus $\mathrm{J}$ is also path-independent.

\section{Effect of Electric Fields on Piezoelectric Facture}

6.1 Under purely electric loading of $D_{2}^{\infty}$ only

In this case, the second equations of (25) and (23) give

$$
\Pi_{2 \infty}=\mathbf{i}_{4}\left(D_{2}^{\infty}-D_{2}^{0}\right), D_{2}^{\infty}-D_{2}^{0}=\frac{\lambda}{1+\lambda} D_{2}^{\infty},
$$

respectively. Equations (74) and (78) can be written as

$$
\begin{array}{r}
J_{l}=\frac{\pi a}{4} \mathbf{i}_{4}^{T} \mathbf{H i}_{4}\left(D_{2}^{\infty}-D_{2}^{0}\right)^{2}, \\
=\frac{\pi a}{4} H_{44} \frac{\lambda^{2}}{(1+\lambda)^{2}}\left(D_{2}^{\infty}\right)^{2}, \\
J_{c s}=\frac{\pi a}{4} \mathbf{i}_{4}^{T} \mathbf{H i}_{4}\left(D_{2}^{\infty}-D_{2}^{0}\right) D_{2}^{0}, \\
=\frac{\pi a}{4} H_{44} \frac{\lambda}{(1+\lambda)^{2}}\left(D_{2}^{\infty}\right)^{2} .
\end{array}
$$


Substituting (94) and (95) into (80) results in

$$
G=\frac{\pi a}{4} H_{44} \frac{\lambda}{1+\lambda}\left(D_{2}^{\infty}\right)^{2}
$$

Since $H_{44}<0$, one has $G<0$, which implies that the crack always tends to close when an electric load is solely applied.

For a permeable crack, $\lambda=0$,

$$
G=0
$$

and for an impermeable crack, $\lambda \rightarrow \infty, G$ becomes

$$
G=\frac{\pi a}{4} H_{44}\left(D_{2}^{\infty}\right)^{2}<0
$$

Note that (97) and (98) are the results from linear electro-elasticity, which predict that an electric load alone cannot propagate an electrically insulated crack.

The value of $G$ from (96) is closer to that of (97) for $0 \leq \lambda<1$, and hence the crack behaves more like a permeable slit. When $\lambda>1, G$ is closer to that of (98), which means that the crack behaves more like an impermeable slit. The variation of $G$ with $\lambda$ is shown in Fig. 5. Since $\delta_{0} / a$ is small compared to $-Y_{44} \varepsilon_{0}$ for most of crack problems in engineering, which gives $\lambda<1$, the permeable crack model may be more appropriate than the impermeable one. On the other hand, the impermeable boundary conditions are more appropriate for electrically insulated cavities, when $\lambda>1$. Also from (94) and (95), under purely electrical loading, $J_{c s}$ predominates in the global energy release rate when $\lambda<1$, while $J_{l}$ predominates for $\lambda>1$. 


\subsection{Under combined mechanical and electrical loading of $\sigma_{2}^{\infty}$ and $D_{2}^{\infty}$}

In this case, we have from the second equations of (25) and (23) that

$$
\Pi_{2 \infty}=\mathbf{i}_{2} \sigma_{2}^{\infty}+\mathbf{i}_{4}\left(D_{2}^{\infty}-D_{2}^{0}\right), D_{2}^{\infty}-D_{2}^{0}=\frac{\lambda}{1+\lambda} D_{2}^{\infty}-\frac{1}{1+\lambda} \frac{H_{42}}{H_{44}} \sigma_{2}^{\infty},
$$

respectively. Equation (61) can be written as

$$
G=\frac{\pi a}{4}\left[\mathbf{i}_{2} \sigma_{2}^{\infty}+\mathbf{i}_{4}\left(D_{2}^{\infty}-D_{2}^{0}\right)\right]^{T} \mathbf{H}\left[\mathbf{i}_{2} \sigma_{2}^{\infty}+\mathbf{i}_{4}\left(D_{2}^{\infty}-D_{2}^{0}\right)\right]+\frac{\pi a}{4} \mathbf{i}_{4}^{T} \mathbf{H}\left[\dot{i}_{2} \sigma_{2}^{\infty}+\mathbf{i}_{4}\left(D_{2}^{\infty}-D_{2}^{0}\right)\right] D_{2}^{0},
$$

where $\mathbf{i}_{2}=(0,1,0,0)^{T}$. A substitution of (99) into (100) yields

$$
G=\frac{\pi a}{4} \hat{H}_{22}\left(\sigma_{2}^{\infty}\right)^{2}+\frac{\pi a}{4} H_{44} \frac{\lambda}{1+\lambda}\left(D_{2}^{\infty}\right)^{2}+\frac{\pi a}{2} H_{42} \frac{\lambda}{1+\lambda} D_{2}^{\infty} \sigma_{2}^{\infty} .
$$

where

$$
\hat{H}_{22}=H_{22}-\frac{H_{42}^{2}}{H_{44}}>0
$$

Equation (101) can be rewritten as

$$
\hat{G}=\hat{H}_{22}\left(\sigma_{2}^{\infty}\right)^{2}+\hat{\lambda} H_{44}\left(D_{2}^{\infty}\right)^{2}+2 \hat{\lambda} H_{24} D_{2}^{\infty} \sigma_{2}^{\infty},
$$

where

$$
\hat{G}=\frac{4 G}{\pi a}, \hat{\lambda}=\frac{\lambda}{1+\lambda} .
$$

Form (102) and $\hat{H}_{22}>0$, we have that for a given value of $D_{2}^{\infty}, \hat{G}$ is positive if the mechanical load $\sigma_{2}^{\infty}$ exceeds a critical value

$$
\sigma_{2 c}^{\infty}=\frac{D_{2}^{\infty}}{\hat{H}_{22}}\left[\sqrt{\left(\hat{\lambda} H_{24}\right)^{2}+\hat{\lambda} \hat{H}_{22}\left(-H_{44}\right)}-\hat{\lambda} H_{24}\right]
$$

When $\sigma_{2}^{\infty}$ is below the critical value, $\hat{G}$ is negative. Equation (103) shows that for permeable cracks, $\hat{\lambda}=0$, thus $\sigma_{2 c}^{\infty}=0$; and for impermeable cracks $\hat{\lambda} \rightarrow 1$, the critical mechanical load is 


$$
\sigma_{2 c}^{\infty}=\frac{D_{2}^{\infty}}{\hat{H}_{22}}\left[\sqrt{\left(H_{24}\right)^{2}+\hat{H}_{22}\left(-H_{44}\right)}-H_{24}\right]
$$

For a given value of $\sigma_{2}^{\infty}$, the variation of the energy release rate $v s$. the electric loading is shown in Fig. 6 for different values of $H_{24}$, where $\left[D_{2}^{\infty}\right]_{1},\left[D_{2}^{\infty}\right]_{2},\left[D_{2}^{\infty}\right]_{0}$ and $d_{0}$ are, respectively, given by

$$
\begin{array}{r}
{\left[D_{2}^{\infty}\right]_{1}=\frac{\sigma_{2}^{\infty}}{\hat{\lambda}\left(-H_{44}\right)}\left[\hat{\lambda} H_{24}-\sqrt{\left(\hat{\lambda} H_{24}\right)^{2}+\hat{\lambda} \hat{H}_{22}\left(-H_{44}\right)}\right],} \\
{\left[D_{2}^{\infty}\right]_{2}=\frac{\sigma_{2}^{\infty}}{\hat{\lambda}\left(-H_{44}\right)}\left[\hat{\lambda} H_{24}+\sqrt{\left(\hat{\lambda} H_{24}\right)^{2}+\hat{\lambda} \hat{H}_{22}\left(-H_{44}\right)}\right],} \\
{\left[D_{2}^{\infty}\right]_{0}=\sigma_{2}^{\infty} \sqrt{\frac{\hat{H}_{22}}{-H_{44}} \hat{\lambda}}, d_{0}=\frac{H_{24}}{-H_{44}} \sigma_{2}^{\infty} .}
\end{array}
$$

For a poled ceramic with the poling direction being along the positive (or negative) $x_{2}$ axis, $H_{24}>0$ (or $H_{24}<0$ ), while $H_{24}=0$ for a depoled ceramic. The effects of positive and negative electric loadings on the energy release rate are shown in Fig. 6. These effects are mechanical-loaddependent.

\section{Concluding Remarks}

The energy release rate of an electrically insulated crack has been derived based on the energy equilibrium principle. We find that the energy release rate $G$ does not equal to the J-integral. In general, $G$ equals to the sum of the local J-integral around the crack tip and the average contribution from the electric field along the entire crack surface. Under purely electric loading, the energy release rate is always negative, indicating that the crack has a tendency to close. Under combined 
mechanical and electrical loading, the energy release rate is positive when the applied mechanical load exceeds a critical value, which is a function of the strength of the applied electrical field. An electric field may decrease or increase the energy release rate depending on the strength of the electric field and the level of the mechanical load. The effect of electric field on the fracture behaviour of piezoelectric media is thus mechanical-load-dependent.

It should be pointed out that the present work is based on linear electro-elasticity. During the failure process of a piezoelectric material, the material, especially at the vicinity of a crack, behaves nonlinearly. Thus, the failure behavior of piezoelectric materials might deviate from the predictions based on linear approaches. Even so, the linear results are still foundational and important to the development of nonlinear analyses. Moreover, the linear results might be also used to predict the fracture behavior of piezoelectric materials at low levels of loading, at which the nonlinear behavior might occur at a very small crack-processing zone and thus, the materials could reveal a macroscopically linear fracture behavior.

\section{Acknowledgments}

This work was supported by a grant (HKUST6039/02E) from the Research Grants Council of the Hong Kong Special Administrative Region, China. TYZ thanks the Croucher Foundation for the Croucher Senior Research Fellowship Award, which gave him more research time by releasing him from teaching duties. 


\section{References}

[1] V.Z. Parton, Fracture Mechanics of Piezoelectric Materials, Acta Astronaut.3 (1976) 671-683.

[2] W.F.J. Deeg, The Analysis of Dislocation, Crack, and Inclusion Problems in Piezoelectric Solids. Ph.D. Thesis, Stanford University, Stanford, California, 1980.

[3] Y.E. Pak, Crack Extension Force in a Piezoelectric Material, ASME J. Appl. Mech.57 (1990) 647-653.

[4] H.A.Sosa, Three-Dimensional Eigenfunction Analysis of a Crack in a Piezoelectric Material, Int. J. Solids Struct. 26 (1990) 1-15.

[5] T.Y.Zhang, J.E. Hack, Mode III Cracks in Piezoelectric Materials, J. Appl. Phys.71 (1992) 5865-5870.

[6] Z. Suo, C.M. Kuo, D.M. Barnett, J.R.Willis, Fracture mechanics for piezoelectric ceramics, J. Mech. Phys. Solids 40 (1992) 739-765.

[7] S.B.Park, C.T. Sun, Effect of electric field on fracture of piezoelectric ceramics, Int. J. Fract.70 (1995) 203-216.

[8] H.Gao, D.M.Barnett, An Invariance Property of Local Energy Release Rates in a Strip Saturation Model of Piezoelectric Fracture, Int. J. Fract. 79 (1996) R25-R29.

[9] T.Y.Zhang, P.Tong, Fracture Mechanics for a Mode III Crack in a Piezoelectric Material, Int. J. Solids Struct. 33 (1996) 343-359.

[10] T.Y.Zhang, C.F. Qian, P. Tong, Linear Electro-Elastic Analysis of a Cavity or a Crack in a Piezoelectric Material, Int. J. Solids Struct. 35 (1998) 2121-2149.

[11] C.Q.Ru, Electric-Field Induced Crack Closure in Linear Piezoelectric Media, Acta Meter 47 (1999) 4683-4693.

[12] C.F.Gao, W.X. Fan, Exact Solutions for the Plane Problem in Piezoelectric Media with an Elliptic Hole or a Crack, Int. J. Solids Struct.36 (1999) 2527-2540.

[13] R.M. McMeeking, Creak Tip Energy Release Rate For a Piezoelectric Compact Tension Specimen, Eng. Fract. Mech. 64 (1999) 217-244. 
[14] R.M.McMeeking, Towards a Fracture Mechanics For Brittle Piezoelectric and Dielectric Materials, Int. J. Fract.108 (2001) 25-41.

[15] T.Y.Zhang, M.H.Zhao, P. Tong, Fracture of Piezoelectric Ceramics, Adv. Appl. Mech. 38 (2001) 148289.

[16] R.M.McMeeking, Electrostrictive forces near crack like flaws, J. Appl. Math. Phsy.40 (1989) 615-627.

[17] T.Y.Zhang, Effect of sample width on the energy release rate and electric boundary conditions along crack surface in piezoelectric materials, Int. J. Fracture 66 (1994) R33-38.

[18] S.Li, On Global Energy Release Rate of a Permeable Crack in Piezoelectric Ceramic, ASME J. Appl. Mech., 70 (2003) 246-252.

[19] R.M. McMeeking, The Energy Release Rate For a Griffith Crack in a Piezoelectric Material, Eng. Fract. Mech. (2003) in press.

[20] G.A.Schneider, F. Felten, R.M. McMeeking, The Electrical Potential Difference Across Cracks in PZT Measured by Kelvin Probe Microscopy and the Implications for Fracture, Acta Meter 51 (2003) 22352241.

[21] D.M.Barnett, J.Lothe, Dislocations and line charges in anisotropic piezoelectric insulators, Phys. Stat. Sol. B-Basic Research, 67 (1997) 105-111.

[22] C.F.Gao, Further Study of the Generalized 2D Problem of an Elliptical Hole or a Crack in Piezoelectric Media, Mech. Res. Commu.27 (2002) 429-434.

[23] G.P.Cherepanov, Mechanics of Brittle Fracture, McGraw-Hill, New York, 1979. 


\section{List of Figure Captions}

Fig.1. An elliptical cavity in a piezoelectric solid subjected to remote loadings

Fig.2. A semi-permeable crack in a piezoelectric solid

Fig.3. J-integral contours

Fig.4. Driving force of electric field on the crack surface

Fig.5. Energy release rate under purely electric loading

Fig.6. Energy release rate under combined mechanical-electric loadings 
Fig.1. An elliptical cavity in a piezoelectric solid subjected to remote loadings

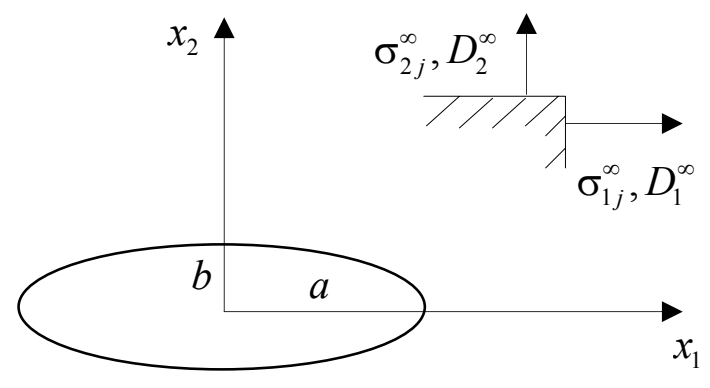


Fig.2. A semi-permeable crack in a piezoelectric solid

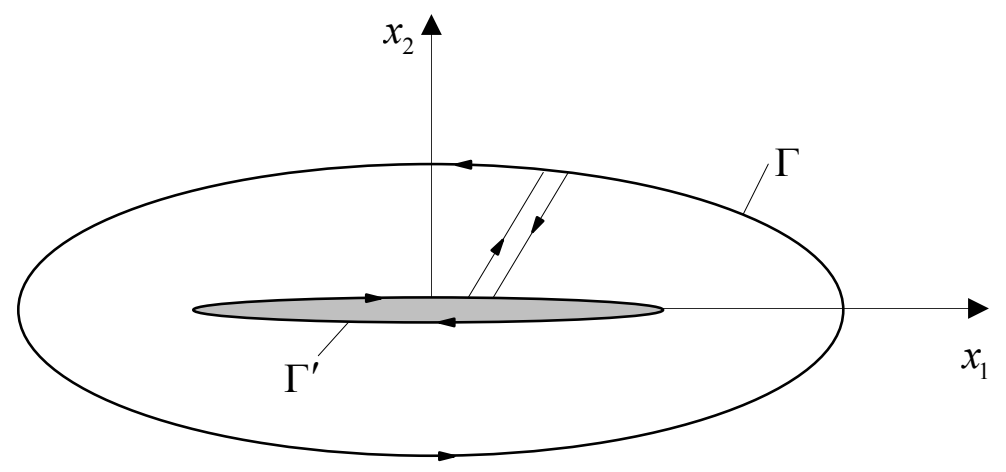


Fig.3. J-integral contours

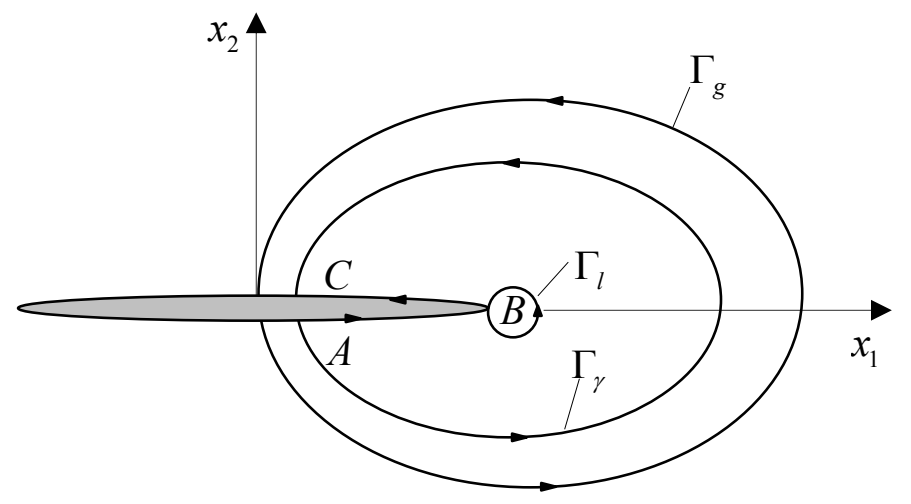


Fig.4. Driving force of electric field on the crack surface

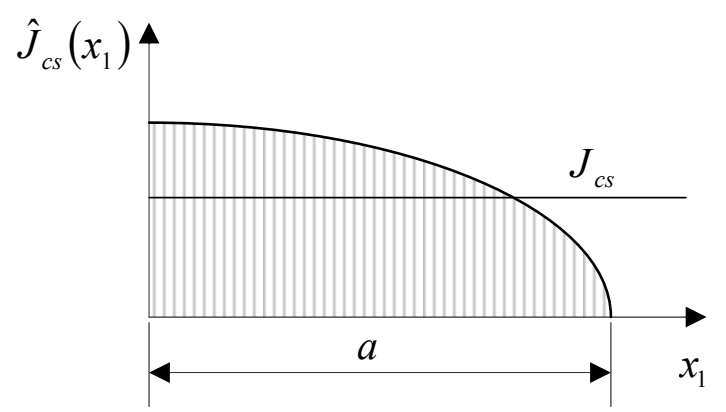


Fig.5 Energy release rate under purely electric loading

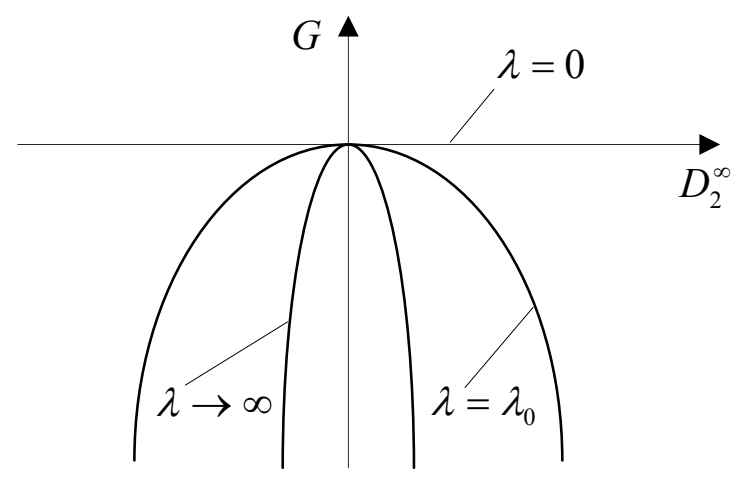


Fig.6 Energy release rate under combined mechanical-electric loadings

(a) $H_{24}>0$

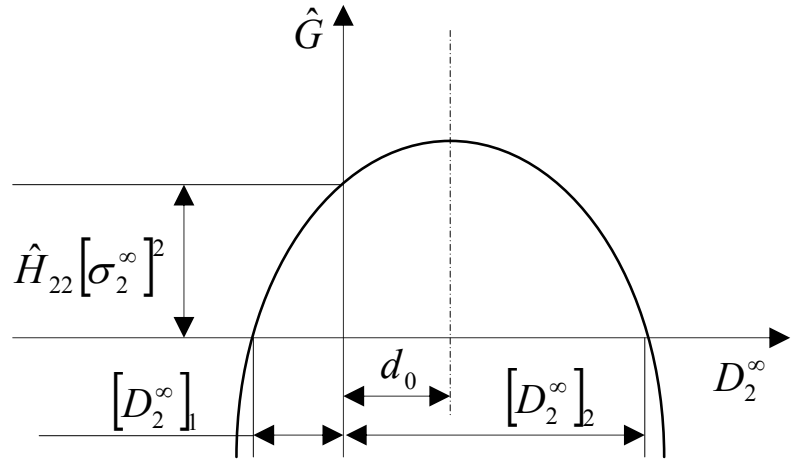

(b) $H_{24}=0$

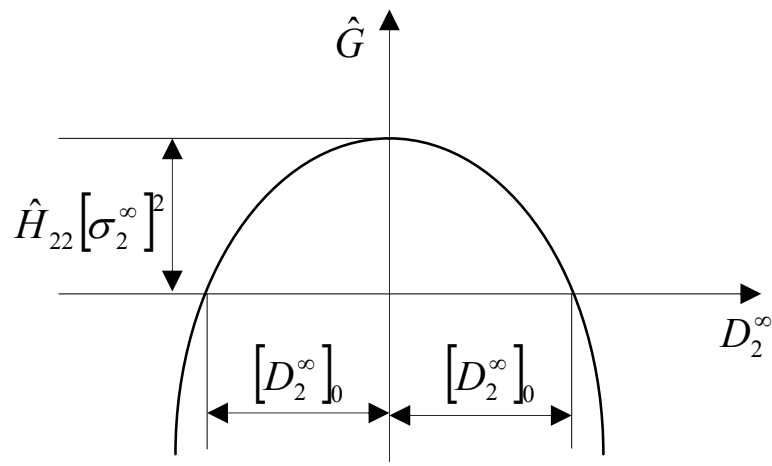

(c) $H_{24}<0$

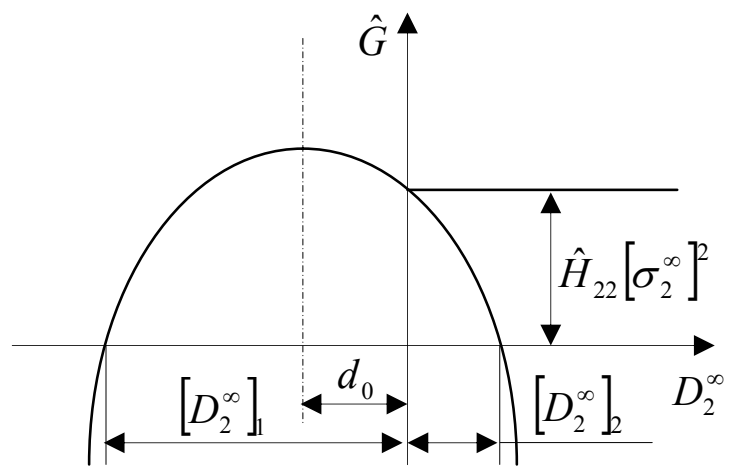

